\title{
Clinical and Molecular Study of 320 Children With Marfan Syndrome and Related Type I Fibrillinopathies in a Series of 1009 Probands With Pathogenic FBN1 Mutations
}

\author{
Laurence Faivre, MD, PhDa,b, Alice Masurel-Paulet, MDa, Gwenaëlle Collod-Béroud, PhD c,d, Bert L. Callewaert, MDe, Anne H. Child, MD, FRCPf, \\ Chantal Stheneur, MD, PhDg, Christine Binquet, MD, PhD ${ }^{b}$,h , Elodie Gautier, MSc ${ }^{b, h}$, Bertrand Chevallier, MDg, Frédéric Huet, MD, PhDa, \\ Bart L. Loeys, MD, PhDe,i,j, Eloisa Arbustini, MDk', Karin Mayer, PhD', Mine Arslan-Kirchner, MDm, Anatoli Kiotsekoglou, MDg, \\ Paolo Comeglio, PhDg, Maurizia Grasso, PhDk, Dorothy J. Halliday, BSc, MBBS, MRCP, PhD n , Christophe Béroud, MD, PhDc,d,o, \\ Claire Bonithon-Kopp, MD, PhD ${ }^{b, h}$, Mireille Claustres, MD, PhDc,d,o, Peter N. Robinson, MD, MSc ${ }^{p}$, Lesley Adès, MBBS, FRACP, MD, CGq,r,s, \\ Julie De Backer, MD, PhDe, Paul Coucke, PhDe, Uta Francke, MD, PhD ${ }^{t}$, Anne De Paepe, MD, PhDe, Catherine Boileau, PharmD, PhDu, \\ Guillaume Jondeau, MD, PhDv
}

\begin{abstract}
aGenetic Center and bCenter of Clinical Investigation, Epidemiological/Clinical Trials, Centre Hospitalier Universitaire Dijon, Dijon, France; 'Institut National de la Santé et de la Recherche Médicale (INSERM) U827, Montpellier, France; 'Montpellier University I, Montpellier, France; eCenter for Medical Genetics, Ghent University Hospital, Ghent, Belgium; fDepartment of Cardiological Sciences, St George's Hospital, London, England; 9Pediatric Service and uLaboratory of Molecular Genetics, Ambroise Paré Hospital, Assistance Publique-Hôpitaux de Paris, Université Versailles-Saint Quentin en Yvelines, Boulogne, France; hInstitut National de la Santé et de la Recherche Médicale Centre d'Investigation Epidémiologique, Dijon, France; IInstitute of Genetic Medicine and the jHoward Hughes Medical Institute, School of Medicine, Johns Hopkins University, Baltimore, Maryland; kCenter for Inherited Cardiovascular Diseases, Foundation Instituto di Ricovero e Cura a Carattere Scientifico Policlinico San Matteo, Pavia, Italy; 'Center for Human Genetics and Laboratory Medicine, Martinsried, Germany; mInstitute for Human Genetics, Hannover, Germany; nepartment of Biochemistry, University of Oxford, Oxford, England; 'Centre Hospitalier Universitaire Montpellier, Laboratory of Molecular Genetics, Arnault de Villeneuve Hospital, Montpellier, France; PInsitute for Genetic Medicine, Universitätsmedizin Charité, Berlin, Germany; aMarfan Research Group and 'Department of Clinical Genetics,

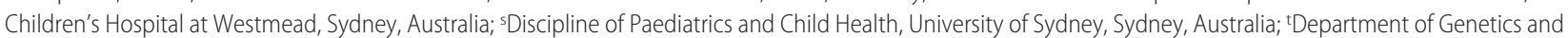
Pediatrics, Stanford University Medical Center, Stanford, California; 'Multidisciplinary Marfan Consultation, Bichat Hospital, AP-HP, Paris, France
\end{abstract}

The authors have indicated they have no financial relationships relevant to this article to disclose.

\section{ABSTRACT}

From a large series of 1009 probands with pathogenic FBN1 mutations, data for 320 patients $<18$ years of age at the last follow-up evaluation were analyzed $(32 \%)$. At the time of diagnosis, the median age was 6.5 years. At the last examination, the population was classified as follows: neonatal Marfan syndrome, $14 \%$; severe Marfan syndrome, 19\%; classic Marfan syndrome, 32\%; probable Marfan syndrome, 35\%. Seventy-one percent had ascending aortic dilation, 55\% ectopia lentis, and $28 \%$ major skeletal system involvement. Even when aortic complications existed in childhood, the rates of aortic surgery and aortic dissection remained low $(5 \%$ and $1 \%$, respectively). Some diagnostic features (major skeletal system involvement, striae, dural ectasia, and family history) were more frequent in the 10- to $<18$-year age group, whereas others (ascending aortic dilation and mitral abnormalities) were more frequent in the population with neonatal Marfan syndrome. Only 56\% of children could be classified as having Marfan syndrome, according to international criteria, at their last follow-up evaluation when the presence of a FBNI mutation was not considered as a major feature, with increasing frequency in the older age groups. Eighty-five percent of child probands fulfilled international criteria after molecular studies, which indicates that the discovery of a FBNI mutation can be a valuable diagnostic aid in uncertain cases. The distributions of mutation types and locations in this pediatric series revealed large proportions of probands carrying mutations located in exons 24 to $32(33 \%)$ and in-frame mutations $(75 \%)$. Apart from lethal neonatal Marfan syndrome, we confirm that the majority of clinical manifestations

Key Words

Marfan syndrome, FBN1, childhood, international criteria

\section{Abbreviations}

AAD-ascending aortic dilation

$\mathrm{Cl}$ - confidence interval

EL-ectopia lentis

MFS - Marfan syndrome

PTC - premature termination codon

Address correspondence to Laurence Faivre

MD, PhD, Centre de Génétique, Hôpital d'Enfants, 10, bd Maréchal De Lattre de Tassigny, 21034 Dijon, France. E-mail: laurence.faivre@chu-dijon.fr

American Academy of Pediatrics of Marfan syndrome increase with age, which emphasizes the poor applicability of the international criteria to this diagnosis in childhood and the need for follow-up monitoring in cases of clinical suspicion of Marfan syndrome.

$\mathrm{M}$ ARFAN SYNDROME (MFS) (MIM 154700) is a connective tissue disorder with autosomal dominant inheritance that has a prevalence of 1 case per 5000 to 10000 individuals $^{1}$ and is caused mainly by FBN1 mutations..$^{2,3}$ The cardinal features of MFS involve the ocular, cardiovascular, and skeletal systems. ${ }^{4}$ The skin, lung, and dura also may be involved. ${ }^{5}$ In addition to classic MFS, FBN1 mutations are responsible for a large phenotypic spectrum ranging 
from neonatal MFS to mild clinical entities. ${ }^{6}$ Because of the high population frequency and the nonspecific nature of many of the clinical findings in MFS, clinical diagnostic criteria have been established, that is, the Ghent criteria, ${ }^{7}$ which have superseded the previous Berlin criteria. $^{8}$

The phenotype in adults is well documented, and diagnosis of MFS in the context of classic multisystem involvement is relatively straightforward. ${ }^{9-11}$ Diagnosis of MFS in childhood may be problematic, however, because many manifestations are age-dependent, which diminishes the utility of the international criteria. ${ }^{7}$ The marked phenotypic heterogeneity both between and within families and the high incidence of sporadic cases also contribute to the diagnostic difficulties. From a large, collaborative, international study including 1009 probands, all carrying a pathogenic FBN1 mutation, we analyzed the clinical and molecular characteristics of a pediatric subgroup of probands who were $<18$ years of age at their last follow-up evaluation. Our results provide a better description of MFS in childhood and the presenting features at different ages.

\section{METHODS}

A total of 1191 probands carrying a FBN1 mutation who were registered between 1995 and 2005 were identified through the Universal Marfan database-FBN1 (www. umd.be $)^{2,12}$ and the network of participating centers. Inclusion criteria in the present study were (1) heterozygosity for a pathogenic FBN1 gene mutation and (2) the availability of clinical information. Of 1191 probands with a FBNI mutation, 182 probands were excluded from the study (no clinical data available, $n=129$; insufficient data on cardiovascular, ocular, or skeletal involvement, $n=44$; autosomal dominant WeillMarchesani syndrome, $n=4 ; 2$ FBN1 mutations on the same allele, $n=4$; compound heterozygosity, $n=1$ ). The pathogenic nature of a putative mutation was assessed by using recognized criteria. In brief, all nonsense mutations, deletions, and insertions (in or out of frame) were considered pathogenic; for all splice mutations, the wild-type and mutant strength values of the splice sites were compared by using genetic algorithms, and only mutations displaying significant deviation from the normal value were included. Missense mutations were considered pathogenic when $\geq 1$ of the following features was found: (1) de novo missense mutation; (2) missense mutation substituting or creating a cysteine; (3) missense mutation involving a consensus calcium-binding residue; (4) substitution of glycines implicated in correct domain-domain packing; or (5) intrafamilial segregation of a missense mutation involving a conserved amino acid. ${ }^{13}$ The patients included in the study originated from 38 countries, on 5 continents. The clinical data were collected either from a questionnaire sent to referring physicians and clinical investigators or from previous publications in which sufficient information was available. The clinical information included a range of qualitative and quantitative clinical parameters, including the age at the diagnosis of MFS or another type I fibrillinopathy and the presence or absence of clinical fea- tures, including cardiovascular, ocular, skeletal, cutaneous, pulmonary, and dural manifestations.

Data for 320 probands ( $32 \%$ ) who were $<18$ years of age at the last follow-up evaluation were extracted for the study. To describe this population, the frequency of each specific clinical feature or system involvement was studied in the overall population of children and then in 3 subgroups, that is, neonates, children $<10$ years of age at the last follow-up evaluation, and children 10 to $<18$ years of age at the last follow-up evaluation. The clinical features studied in each system are listed in Table 1. The ages at diagnosis and at surgery were collected for scoliosis, ectopia lentis (EL), ascending aortic dilation (AAD), and aortic dissection. For each child, major and/or minor system features were defined for skeletal, ocular, cardiovascular, pulmonary, cutaneous, and dural manifestations, according to the Ghent criteria. ${ }^{7}$ Ghent criteria were defined as positive for isolated cases that presented with 2 major system manifestations and involvement of $\geq 1$ other organ system. In familial cases, the presence of a major criterion finding in 1 organ system and the involvement of a second organ system were sufficient for the diagnosis of MFS if another family member was diagnosed independently as having MFS. To reproduce the situation that clinicians face in clinical practice, the presence of a FBN1 mutation was not considered as a major feature initially. For the group of patients who did not fulfill clinical international criteria, we evaluated the proportions of patients who demonstrated positive results for international criteria when the presence of the FBN1 mutation was considered as a major feature for the general population and for the different groups. Because some of the clinical or radiologic features that are part of the international criteria (including protrusio acetabulae, dural ectasia, flat cornea, iris or ciliary muscle hypoplasia, dilation of the pulmonary artery, mitral annular calcification, and apical blebs) were investigated only occasionally, unmentioned clinical features had to be considered absent for determination of patients' status with respect to the international criteria.

The first presenting diagnostic feature was determined for each patient. For descriptive purposes, the overall population was classified as having neonatal MFS (defined in this study as a diagnosis of type I fibrillinopathy with severe valvular involvement before 4 weeks of age), severe MFS (defined by the diagnosis of MFS according to clinical international criteria, including AAD, before 10 years of age), classical MFS (defined by the diagnosis of MFS according to clinical international criteria in other patients), or probable MFS (defined on the basis of unfulfilled clinical international criteria in childhood, ie, before 18 years of age). Mutation types and locations were compared for the different presentation types.

The Kaplan-Meier method ${ }^{14}$ was used to estimate the cumulative probabilities (and their confidence intervals [CIs]) of diagnoses of clinical features at 10 years of age when the ages at diagnosis were known (AAD, dissection, and surgery; EL and surgery for EL; and scoliosis). The $\chi^{2}$ test was used to analyze the distribution of mutations (locations and types) according to MFS presentation. Only $P$ 
TABLE 1 Frequency of Clinical Features of the Ghent Criteria for the 320 Child Probands

\begin{tabular}{|c|c|c|c|c|}
\hline \multirow[t]{2}{*}{ Clinical Features } & \multicolumn{4}{|c|}{ Frequency, \% } \\
\hline & $\begin{array}{c}\text { Total } \\
(N=320)\end{array}$ & $\begin{array}{l}\text { Neonatal MFS } \\
(N=45)\end{array}$ & $\begin{array}{c}<10 y \\
(N=111)^{a}\end{array}$ & $\begin{array}{c}10 \text { to }<18 y \\
(N=164)^{\mathrm{a}} \\
\end{array}$ \\
\hline \multicolumn{5}{|l|}{ Skeletal system } \\
\hline Pectus carinatum or excavatum requiring surgery & 27 & 14 & 26 & 31 \\
\hline Dolichostenomelia & 52 & 41 & 48 & 58 \\
\hline Positive wrist and thumb sign & 83 & 100 & 79 & 81 \\
\hline Scoliosis of $>20^{\circ}$ or spondylolisthesis & 43 & 33 & 35 & 52 \\
\hline Limited elbow extension $\left(<170^{\circ}\right)$ & 21 & 81 & 11 & 12 \\
\hline Pes planus & 48 & 22 & 46 & 55 \\
\hline Protrusio acetabulae & 9 & 0 & 6 & 12 \\
\hline Facial appearance & 58 & 82 & 52 & 55 \\
\hline Joint hypermobility & 67 & 64 & 68 & 67 \\
\hline Moderate pectus excavatum & 32 & 14 & 32 & 31 \\
\hline High arched palate & 70 & 68 & 61 & 73 \\
\hline Major skeletal system involvement & 28 & 24 & 20 & 34 \\
\hline Minor skeletal system involvement & 63 & 69 & 67 & 59 \\
\hline \multicolumn{5}{|l|}{ Ocular system } \\
\hline EL & 55 & 38 & 63 & 55 \\
\hline Myopia & 42 & 31 & 44 & 44 \\
\hline Flat cornea & $\mathrm{Nl}$ & $\mathrm{Nl}$ & $\mathrm{Nl}$ & $\mathrm{Nl}$ \\
\hline Iris or ciliary muscle hypoplasia & $\mathrm{NI}$ & $\mathrm{Nl}$ & $\mathrm{NI}$ & $\mathrm{NI}$ \\
\hline Major ocular system involvement & 55 & 38 & 63 & 55 \\
\hline \multicolumn{5}{|l|}{ Cardiovascular system } \\
\hline$A A D$, with or without aortic regurgitation & 71 & 93 & 66 & 68 \\
\hline Ascending aortic dissection & 1 & 0 & 1 & 1 \\
\hline $\begin{array}{l}\text { Dilation or dissection of descending aorta or } \\
\text { abdominal aorta }\end{array}$ & 2 & 4 & 1 & 2 \\
\hline Mitral valve prolapse & 62 & 73 & 62 & 59 \\
\hline Mitral annulus calcification & $\mathrm{NI}$ & $\mathrm{Nl}$ & $\mathrm{NI}$ & $\mathrm{Nl}$ \\
\hline Dilation of pulmonary artery & $\mathrm{NI}$ & $\mathrm{NI}$ & $\mathrm{NI}$ & $\mathrm{NI}$ \\
\hline Major cardiac system involvement & 71 & 93 & 66 & 68 \\
\hline Minor cardiac system involvement & 14 & 2 & 19 & 15 \\
\hline \multicolumn{5}{|l|}{ Pulmonary system } \\
\hline Pneumothorax or apical blebs & 4 & 7 & 3 & 4 \\
\hline Minor lung system involvement & 4 & 7 & 3 & 4 \\
\hline \multicolumn{5}{|l|}{ Skin } \\
\hline Striae atrophicae & 19 & 2 & 7 & 32 \\
\hline Recurrent or incisional hernia & 7 & 16 & 4 & 7 \\
\hline Minor skin involvement & 23 & 18 & 9 & 34 \\
\hline \multicolumn{5}{|l|}{ Dura } \\
\hline Lumbosacral dural ectasia & 26 & 0 & 11 & 35 \\
\hline Major central nervous system involvement & 5 & 0 & 2 & 9 \\
\hline \multicolumn{5}{|l|}{ Family } \\
\hline $\begin{array}{l}\text { First-degree family member fulfilling diagnostic } \\
\text { criteria }\end{array}$ & 29 & 0 & 22 & 43 \\
\hline Pathogenic FBN1 mutation & 100 & 100 & 100 & 100 \\
\hline Clinical Ghent criteria positive & 56 & 36 & 52 & 64 \\
\hline $\begin{array}{l}\text { Ghent criteria positive after FBN1 mutation } \\
\text { screening }\end{array}$ & 85 & 93 & 80 & 85 \\
\hline
\end{tabular}

values of $<.001$ were considered significant, because multiple tests were performed. Analyses were performed by using Stata 9.0 (Stata Corp, College Station, TX).

\section{RESULTS}

Of the 320 child probands, 55\% were male and $45 \%$ were female. Only 94 patients $(30 \%)$ had a family history of MFS or other type I fibrillinopathy. The median age at diagnosis was 6.5 years (interquartile range: $3-11$ years). Forty-five patients (14\%) had a neonatal MFS presentation. Of the remaining patients, 111 (35\%) were $<10$ years of age at their last follow-up evaluation and $164(51 \%)$ were between 10 and 18 years of age. Thirty-six children $(11 \%)$ were dead at the last follow-up evaluation (29 as a result of cardiac failure, including 25 with neonatal MFS; 3 in the context of cardiac surgery, at birth, 4 years, and 16 years; 1 as a result of respiratory insufficiency in the context of severe 


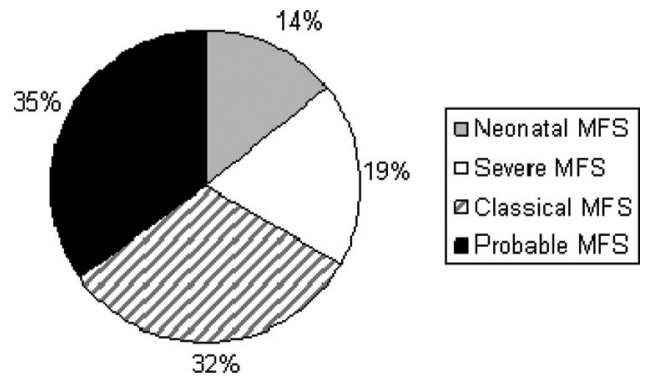

FIGURE 1

Type of MFS presentation in the pediatric population with MFS ( $N=320)$, when the presence of a FBN1 mutation was not taken into account (see "Methods" for definitions of the different types of presentation).

emphysema; 1 as a result of aortic dissection at 16 years of age; 1 as a result of another cause, independent of MFS; and 1 as a result of termination of pregnancy for a fetus with severe prenatally ultrasonographically detected features and asymptomatic parents, which suggested neonatal MFS). The population was classified as follows: neonatal MFS, 14\%; severe MFS, 19\%; classic MFS, 32\%; probable MFS, 35\% (Fig 1). The frequencies of the clinical manifestations that are part of the international criteria are presented in Table 1 for the overall population of probands and the different groups.

Figure 2 shows Kaplan-Meier estimates of the probability of diagnosing an aortic event in this population. The cumulative probability of AAD diagnosed at 10 years was 53\% (99.9\% CI: 44\%-63\%). The probability of aortic surgery was 5\% (99.9\% CI: $1 \%-14 \%)$, and the probability of ascending aortic dissection was $1 \%$ (99.9\% CI: 0\%-11\%). Aortic surgery was necessary for 14 probands with severe aortic dilation and 3 probands with aortic dissection. Of the 3 patients with ascending aortic dissections, at 8, 15, and 16 years of age, 2 patients also had involvement of the descending and/or abdominal aorta. The cumulative probabilities of mitral valve

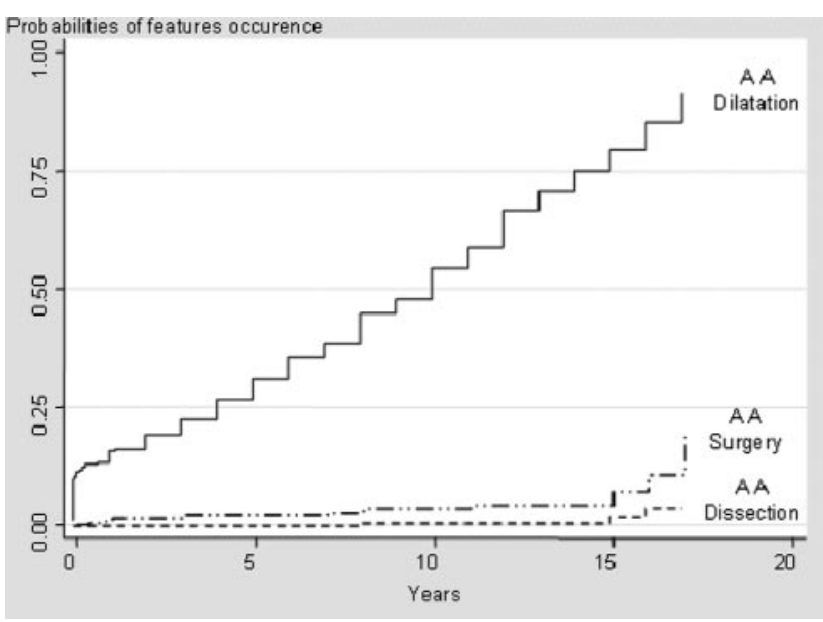

FIGURE 2

Kaplan-Meier analysis of the probability of diagnosing an aortic event in the pediatric population with MFS $(N=320)$. The probabilities of ascending aortic surgery and ascending aortic dissection were determined in the overall population of child probands. AA indicates ascending aortic. prolapse, mitral insufficiency, aortic insufficiency and valvular surgery independent of aortic surgery at 10 years were 49\% (99.9\% CI: 40\%-59\%), 34\% (99.9\% CI: $25 \%-44 \%), 10 \%(99.9 \%$ CI: $5 \%-18 \%)$, and $6 \%$ (99.9\% CI: $2 \%-13 \%)$, respectively. Congenital structural heart disease was found for 6 probands. When results were stratified for the different groups, the frequencies of AAD and valvular abnormalities were higher among those with neonatal MFS (Table 1).

With respect to ocular manifestations, the cumulative probability of EL diagnosed at $\leq 10$ years was $52 \%$ $(99.9 \%$ CI: $42 \%-62 \%)$ and that for eye surgery was $24 \%(99.9 \%$ CI: $14 \%-39 \%)$. With stratification into different age groups, the frequency of EL was higher in the $<10$-year age group $(63 \%)$ and lower in the neonatal MFS group (Table 1). Myopia was diagnosed for $42 \%$ of patients, whereas cataracts, retinal detachment, and glaucoma each were diagnosed for $1 \%$ of children.

With respect to skeletal manifestations, $28 \%$ of probands had major skeletal system involvement and $63 \%$ had minor skeletal system involvement at their last follow-up evaluation. The cumulative probability of scoliosis diagnosed at $\leq 10$ years was $47 \%$ (99.9\% CI: $37 \%-$ $59 \%)$. Orthopedic surgery was performed for $8 \%$ of probands (scoliosis, $n=14$; pectus carinatum or excavatum, $n=5$; other indications, $n=8$ ). One hundred five $(52 \%)$ of 203 probands had heights $>3$ SDs above the mean. Three probands had craniosynostosis. The frequencies of most skeletal features (pectus carinatum or excavatum requiring surgery, dolichostenomelia, scoliosis, pes planus, and protrusio acetabulae) increased with age (Table 1).

With respect to other manifestations, the proportions of patients with striae, dural ectasia, and family history increased with age (Table 1). A diaphragmatic hernia was noted for 7 patients with neonatal MFS. Hydrocephalus was described in 1 case of neonatal MFS. Other features (polymicrogyria, mitochondrial complex I deficiency, and epidermolysis bullosa) were described only once, which indicates a coincidental association.

As expected for the general population, ${ }^{15} 10$ probands $(3 \%)$ had developmental delay/mental retardation. The presence of mental retardation could be considered coincidental for 3 patients, because 1 patient also had mitochondrial complex I deficiency and 2 patients had a family history of MFS without mental retardation. Mental retardation also was described for 2 patients with neonatal MFS and long survival times ( 3 and 4 years) and 1 patient with craniosynostosis reported as Shprintzen-Goldberg syndrome. The 4 remaining patients represented sporadic cases or had unknown family history, with presentation as severe MFS in 3 cases and classic MFS in 1 case. The patients with polymicrogyria and hydrocephalus were not included in the number of patients with developmental delay/mental retardation because the patients died at 1 day and 2 months of life.

The majority $(85 \%)$ of patients could be classified as having MFS according to international criteria at their last follow-up evaluation when the presence of a FBN1 mutation was considered as a major feature, but only $56 \%$ of the children met the clinical international criteria when the 
TABLE 2 First Diagnostic Manifestation in the Overall Population of Child Probands and in the Different Age Groups at the Time of MFS (or Other Type I Fibrillinopathy) Diagnosis

\begin{tabular}{lccc}
\hline $\begin{array}{c}\text { First Diagnostic } \\
\text { Manifestation }\end{array}$ & \multicolumn{3}{c}{ Proportion, \% } \\
\cline { 2 - 4 } & $\begin{array}{c}\text { Total } \\
(N=320)\end{array}$ & $\begin{array}{c}<10 y \\
(N=166)\end{array}$ & $\begin{array}{c}10 \text { to }<18 \text { y } \\
(N=109)\end{array}$ \\
\hline EL + AAD & 29 & 30 & 34 \\
AAD & 27 & 19 & 30 \\
Skeletal & 24 & 25 & 20 \\
EL & 20 & 27 & 16 \\
\hline
\end{tabular}

presence of a FBNI mutation was not considered as a major feature. When the populations of children in the different age groups were considered, $36 \%$ in the neonatal MFS group, $52 \%$ in the $<10$-year group, and $64 \%$ in the 10 - to $<18$-year group met the clinical international criteria (Table 1). Table 1 also shows the proportion of patients who could be diagnosed as having MFS when the presence of the FBN1 mutation was considered. Of the patients with ascending aortic dissection or surgery, 4 (24\%) did not fulfill the clinical international criteria.

Table 2 shows that the first diagnostic features leading to the diagnosis of MFS were divided fairly evenly between AAD, EL, both EL and AAD, and skeletal involvement in the overall population of child probands. Results also are presented according to the age at diagnosis.

The distribution of mutations in the proband cohort was as follows: 232 mutations (75\%) were in-frame mutations, whereas $78(25 \%)$ generated a premature termination codon (PTC). Ten splice site mutations were not classified because the consequences at the mRNA level could not be determined unambiguously. Within the missense mutations, $130(66 \%)$ of 196 involved a cysteine ( 111 replacing and 19 creating a cysteine). Most mutations $(79 \% ; n=252)$ were located in an epidermal growth factor domain, 241 (75\%) were in a calciumbinding epidermal growth factor domain, and 38 (12\%) were in a transforming growth factor $\beta_{1}$-binding protein domain. One hundred four mutations (33\%) were lo- cated in exons 24 to 32 . A $\chi^{2}$ analysis for the distribution of mutations according to the type of presentation revealed significant differences for children with mutations in exons 24 to 32, compared with children with mutations located elsewhere $(P<.001)$ (Fig 3). Indeed, a larger proportion of mutations in exons 24 to 32 was found for patients with a neonatal presentation, compared with children with classic or probable MFS. Two of the 3 probands who developed aortic dissection and 9 of the 14 probands who underwent surgery because of aortic dilation had mutations in the exon 24 to 32 region. Significant results also were found for PTC mutations versus in-frame mutations, with the proportion of PTC mutations decreasing with the severity of the presentation $(P<.001)$ (Fig 3$)$.

\section{DISCUSSION}

The literature description of MFS at pediatric ages is scanty. Previous reports of children with MFS included only small numbers of patients, with a strong bias in clinical presentation. Authors have concentrated mainly on severely affected individuals with prominent cardiovascular involvement. ${ }^{16-21}$ The 2 largest studies included 40 and 52 children. ${ }^{22,23}$ Lipscomb et $\mathrm{al}^{22}$ focused on the evolution of the physical characteristics of MFS throughout childhood. By studying probands and relatives $<16$ years of age who were identified through a regional MFS registry and excluding neonatal MFS, the authors showed that musculoskeletal features predominated in and evolved throughout childhood. The most frequent musculoskeletal findings at presentation were a combination of tall stature, limb disproportion, pectus deformity, classic facial features, high arched palate, and arachnodactyly. Only 15\% of patients had EL, 18\% had mitral valve prolapse, and $43 \%$ had AAD, which usually became evident at $\sim 9$ to 15 years of age. The authors concluded that the absence of aortic root enlargement could not be used to exclude a diagnosis of MFS for a "marfanoid" child before skeletal growth was complete. ${ }^{22}$ Very different cardiovascular values were described in the study by van Karnebeek et al,23 in which
FIGURE 3

Distributions of locations (mutations located in exons 24-32) and types of FBN1 mutations (mutations leading to a PTC) depending on the presentation of MFS or type I fibrillinopathy in the pediatric population with MFS $(N=320)$. ${ }^{\text {a }} \chi^{2}$ test $P$ value.

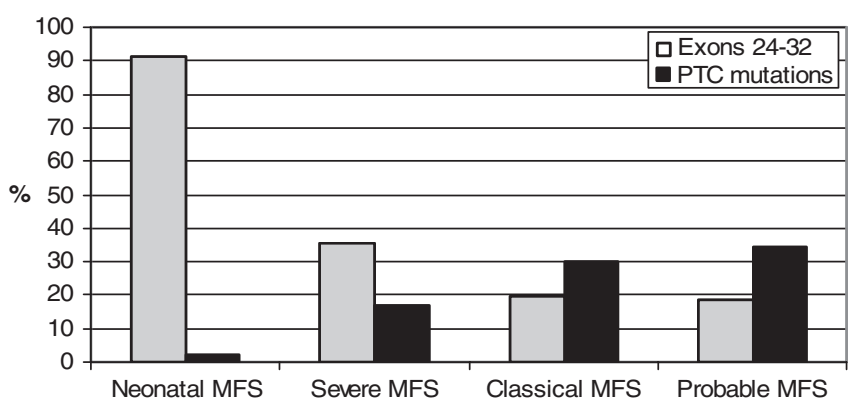

\begin{tabular}{|l|c|c|c|c|}
\hline & $\begin{array}{c}\text { Neonatal MFS vs } \\
\text { other MFS } \\
\text { presentations }\end{array}$ & $\begin{array}{c}\text { Severe MFS vs } \\
\text { other MFS } \\
\text { presentations }\end{array}$ & $\begin{array}{c}\text { Classical MFS vs } \\
\text { other MFS } \\
\text { presentations }\end{array}$ & $\begin{array}{c}\text { Probable MFs vs } \\
\text { other MFS } \\
\text { presentations }\end{array}$ \\
\hline $\begin{array}{l}\text { Exon 24-32 vs other } \\
\text { exons }\end{array}$ & $<.0001^{\mathrm{a}}$ & $.5764^{\mathrm{a}}$ & $.0021^{\mathrm{a}}$ & $.0001^{\mathrm{a}}$ \\
\hline $\begin{array}{l}\text { PTC vs missense } \\
\text { mutations }\end{array}$ & $.0002^{\mathrm{a}}$ & $.1062^{\mathrm{a}}$ & $.1755^{\mathrm{a}}$ & $.0070^{\mathrm{a}}$ \\
\hline
\end{tabular}


52 children who were referred to their pediatric department and fulfilled the international criteria were investigated, with exclusion of patients with neonatal MFS. Eighty-eight percent of those children had mitral valve prolapse, $77 \%$ AAD, $48 \%$ mitral regurgitation, and $25 \%$ aortic regurgitation. Aortic surgery was performed for $23 \%$ of children with AAD (after aortic dissection for 2 patients), and mitral valve surgery was performed for $13 \%$ of patients with mitral valve abnormalities. In addition, $62 \%$ of patients had EL. ${ }^{23}$ The methods for ascertainment and referral of patients might represent the main explanation for these differences.

Our larger sample size, different method of ascertainment through a mutation database, inclusion of only probands, and molecular confirmation of the diagnosis of MFS or type I fibrillinopathy allow a more-accurate description of the organ involvement of MFS at pediatric ages. The large patient number allowed us to stratify the cohort into different groups on the basis of neonatal status and age at last follow-up evaluation, for descriptive and prognostic purposes. We showed that the frequency of AAD was higher in neonatal MFS (Table 1). We also showed that aortic complications were rare in childhood, because aortic dissection occurred in only 3 probands and aortic surgery needed to be performed for 14 patients. As noted in previous publications, ${ }^{16,18,21,24}$ valvular involvement was frequent among the children in our series. The frequency of EL was higher in the $<$ 10-year age group (Table 1). Finally, the frequencies of many skeletal features, dural ectasia, minor cutaneous involvement, and family history increased with age (Table 1). Therefore, MFS in childhood seems to be represented by differing features, depending on the age at diagnosis. An early-onset presentation can be described in de novo cases, with predominantly a severe aortic and mitral valve phenotype and a high mortality rate. When patients are diagnosed between 10 and 18 years of age, their presentation seems to be similar to that of patients with classic MFS, with increasing frequencies of skeletal features, striae, dural ectasia, and family history. Not surprisingly, because $23 \%$ of the series was represented by neonatal or severe MFS, we found a higher rate of de novo mutations than usually described.

Three patients in this series had craniosynostosis, 1 of whom was described previously. ${ }^{25}$ All 3 patients had mutations located in the exon 24 to 32 region. The possible association between MFS and craniosynostosis is important to note, because it can be a confounder in the diagnosis of Loeys-Dietz syndrome or ShprintzenGoldberg syndrome. ${ }^{26-28}$ Only the patient reported by Sood et $\mathrm{al}^{25}$ also had mental retardation. There were 7 patients ( $2 \%$ of the population) with mental retardation that could not be stated as being coincidental. The presence of mental retardation usually turns clinicians to alternative diagnoses, such as Lujan-Fryns syndrome. ${ }^{29}$ We do not provide arguments in favor of an association between MFS and mental retardation because the rate of mental retardation in this series was similar to that in the general population. ${ }^{15}$

We were able to determine the more-frequent diagnostic features at initial presentation in this cohort. In the overall population of children, the rates of the main diagnostic presentations (AAD and EL, AAD, EL, and skeletal features) were quite similar (Table 2). Results did not vary considerably when the population was stratified according to age at the time of diagnosis. However, the retrospective design of our study and the nature of MFS itself preclude accurate definition of the ages at which manifestations arose. Only the age at first detection of a specific feature, which was necessarily older than the true age of occurrence, could be used. Also, these results remain artificial; because the findings of EL and $\mathrm{AAD}$ require additional examinations, the diagnosis is usually triggered by skeletal features.

Results regarding the proportion of patients with positive clinical results for the international criteria confirmed the limited utility of the international criteria in infancy and childhood. Indeed, only $56 \%$ of children would have been diagnosed on clinical grounds alone, compared with $79 \%$ in adulthood. ${ }^{11}$ Although some minor features (eg, mitral valve prolapse, with or without mitral regurgitation, and dysmorphic facial features) were overrepresented in childhood, compared with the adult cohort, the lower rate of positive clinical results for the international criteria in children is explained mainly by the underrepresentation of dural ectasia, minor cutaneous involvement, and family history. ${ }^{11}$ Because only child probands were selected, the absence of a family history is clearly a selection bias. Indeed, milder cases presenting later more frequently have a family history, because the milder mutations permit survival into the reproductive period. Children with new mutations tend to be more seriously affected and may not survive to reproduce. The dura was assessed only occasionally (62 of 320 patients), because evaluation might require anesthesia in young children, access to MRI studies often is limited, and no normal values are readily available. ${ }^{30}$ It is well known that striae usually appear during growth and puberty. ${ }^{5}$ The frequencies of AAD, EL, and major skeletal features were comparable for the child and adult cohorts. ${ }^{10}$ When the population was stratified according to the age at the last follow-up evaluation, we clearly showed that the rate of positive clinical results for the international criteria increased with age (Table 1). Therefore, in cases in which MFS or another type I fibrillinopathy is suspected for a child, one could advocate long-term, clinical, follow-up monitoring in search of developing features suggesting a diagnosis of MFS. Alternatively, because the diagnosis of MFS leads to aortic risk and the identification of a FBN1 mutation leads to the diagnosis of MFS for an additional 29\% of pediatric patients, FBN1 screening could be offered in this population, to limit the risk of loss to follow-up monitoring. It must be emphasized strongly that negative screening results should not indicate discharge of patients from follow-up monitoring.

One limitation of the study could be related to the fact that we selected only child probands. This does not entirely match the clinical situation of caring for children of adult probands, because child probands are more likely to have severe forms of MFS. A FBN1 mutation search as an inclusion criterion might be another bias 
toward severe cases. Nevertheless, our results are intermediate between the results obtained by Lipscomb et $\mathrm{al}^{22}$ whose patients were recruited through a regional registry, and those obtained by van Karnebeek et al, ${ }^{23}$ whose patients were recruited through a pediatric clinic. Also, for determination of the patients' status with respect to the international criteria, we had to consider unmentioned clinical features as absent, which might have biased our results.

In addition to the description of clinical manifestations, we took advantage of our large sample size to study the molecular characteristics of the cohort. Thirtythree percent of mutations were located in the exon 24 to 32 region. This frequency was significantly higher than that in the adult population $(14 \%)^{13}$ and was largely explained by the neonatal MFS cases (Fig 3). In addition, the proportion of mutations leading to PTC in children $(25 \%)$ was significantly smaller than that in the adult cohort $(36 \%)$. This finding may be partly linked to the large proportion of mutations in the exon 24 to 32 region, because we showed previously that PTC mutations were underrepresented in this region. ${ }^{13}$

\section{CONCLUSIONS}

We present a comprehensive clinical and molecular description of a large series of pediatric cases with a FBNI mutation. Besides lethal neonatal MFS, which represents a genuine entity, we confirm that the majority of clinical manifestations of MFS increase with age, highlighting the limited usefulness of international criteria for diagnosis in early infancy and emphasizing the value of FBN1 mutation screening, which not only confirms the diagnosis but also facilitates determination of prognosis and timely management.

\section{ACKNOWLEDGMENTS}

This work was supported by a grant from the French Ministry of Health (grant Programme Hospitalier de Recherche Clinique 2004), Groupement d'Intérêt Scientifique Maladies Rares 2004, Bourse de la Société Francaise de Cardiologie, Fédération Française de Cardiologie 2005, and ANR-05-PCOD-014. Drs Callewaert and Loeys are a research fellow and a senior clinical investigator, respectively, of the Fund for Scientific ResearchFlanders. Drs Child and Comeglio thank the Marfan Trust and the Bluff Field Charitable Fund for support.

We thank H. Plauchu (Lyon, France), D. Halliday (Oxford, United Kingdom), I. Kaitila (Helsinki, Finland), P. Khau Van Kien (Montpellier, France), H. C. Dietz (Baltimore, MD), S. Davies (Cardiff, Wales), and T. Uyeda (Irosaki, Japan) for their participation in the study.

\section{REFERENCES}

1. Pyeritz RE. Marfan syndrome: current and future clinical and genetic management of cardiovascular manifestations. Semin Thorac Cardiovasc Surg. 1993;5(1):11-16

2. Collod-Béroud G, Le Bourdelles S, Adès L, et al. Update of the UMD-FBNl mutation database and creation of an FBN1 polymorphism database. Hum Mutat. 2003;22(3):199-208

3. Dietz HC, Cutting GR, Pyeritz RE, et al. Marfan syndrome caused by a recurrent de novo missense mutation in the fibrillin gene. Nature. 1991;352(6333):337-339

4. Judge DP, Dietz HC. Marfan's syndrome. Lancet. 2005; 366(9501):1965-1976

5. Grahame R, Pyeritz R. The Marfan syndrome: joint and skin manifestations are prevalent and correlated. Br J Rheumatol. 1995;34(2):126-131

6. Robinson PN, Arteaga-Solis E, Baldock C, et al. The molecular genetics of Marfan syndrome and related disorders. J Med Genet. 2006;43(10):769-787

7. De Paepe A, Devereux RB, Dietz HC, Hennekam RC, Pyeritz RE. Revised diagnostic criteria for the Marfan syndrome. Am J Med Genet. 1996;62(4):417-426

8. Beighton P, de Paepe A, Danks D, et al. International Nosology of Heritable Disorders of Connective Tissue, Berlin, 1986. Am J Med Genet. 1988;29(3):581-594

9. Loeys B, Nuytinck L, Delvaux I, De Bie S, De Paepe A. Genotype and phenotype analysis of 171 patients referred for molecular study of the fibrillin-1 gene because of suspected Marfan syndrome. Arch Intern Med. 2001;161(20):2447-2454

10. Child AH, Luitgard N, Robinson PN. Diagnosis and treatment of Marfan syndrome: a summary. In: Robinson PN, Godfrey M, eds. Marfan Syndrome: A Primer for Clinicians and Scientists. New York, NY: Kluwer Publishing/Landes Bioscience; 2004:13-23

11. Faivre L, Collod-Béroud G, Child A, et al. Contribution of molecular analyses in diagnosing Marfan syndrome and type I fibrillinopathies: an international study of 1009 probands. J Med Genet. 2008;45(6):384-390

12. Béroud C, Hamroun D, Collod-Béroud G, Boileau C, Soussi T, Claustres M. UMD (Universal Mutation Database): 2005 update. Hum Mutat. 2005;26(3):184-191

13. Faivre L, Collod-Béroud G, Loeys BL, et al. Effect of mutation type and location on clinical outcome in 1013 probands with Marfan syndrome or related phenotypes with FBN1 mutations: an international study. Am J Hum Genet. 2007;81(3):454-466

14. Kaplan E, Meier P. Nonparametric estimation from incomplete observations. J Am Stat Assoc. 1958;53(282):457-481

15. Roeleveld N, Zielhuis GA, Gabreëls F. The prevalence of mental retardation: a critical review of recent literature. Dev Med Child Neurol. 1997;39(2):125-132

16. Phornphutkul C, Rosenthal A, Nadas AS. Cardiac manifestations of Marfan syndrome in infancy and childhood. Circulation. 1973;47(3):587-596

17. Sisk HE, Zahka KG, Pyeritz RE. The Marfan syndrome in early childhood: analysis of 15 patients diagnosed at less than 4 years of age. Am J Cardiol. 1983;52(3):353-358

18. Geva T, Hegesh J, Frand M. The clinical course and echocardiographic features of Marfan's syndrome in childhood. Am J Dis Child. 1987;141(11):1179-1182

19. Morse RP, Rockenmacher S, Pyeritz RE, et al. Diagnosis and management of infantile Marfan syndrome. Pediatrics. 1990; 86(6):888-895

20. Tayel S, Kurczynski TW, Levine M, et al. Marfanoid children: etiologic heterogeneity and cardiac findings. Am J Dis Child. 1991;145(1):90-93

21. el Habbal MH. Cardiovascular manifestations of Marfan's syndrome in the young. Am Heart J. 1992;123(3):752-757

22. Lipscomb KJ, Clayton-Smith J, Harris R. Evolving phenotype of Marfan's syndrome. Arch Dis Child. 1997;76(1):41-46

23. van Karnebeek CD, Naeff MS, Mulder BJ, Hennekam RC, Offringa M. Natural history of cardiovascular manifestations in Marfan syndrome. Arch Dis Child. 2001;84(2):129-137

24. Pyeritz, RE, Wappel MA. Mitral valve dysfunction in the Marfan syndrome: clinical and echocardiographic study of prevalence and natural history. Am J Med. 1983;74(5):797-807

25. Sood S, Eldadah ZA, Krause WL, McIntosh I, Dietz HC. Muta- 
tion in fibrillin- 1 and the Marfanoid-craniosynostosis (Shprintzen-Goldberg) syndrome. Nat Genet. 1996;12(2):209-211

26. Adès LC, Sullivan K, Biggin A, et al. FBN1, TGFBR1, and the Marfan-craniosynostosis/mental retardation disorders revisited. Am J Med Genet A. 2006;140(10):1047-1058

27. Loeys BL, Schwarze U, Holm T, et al. Aneurysm syndromes caused by mutations in the TGF- $\beta$ receptor. $N$ Engl $J$ Med. 2006;355(8):788-798

28. Shprintzen RJ, Goldberg RB. A recurrent pattern syndrome of craniosynostosis associated with arachnodactyly and abdominal hernias. J Craniofac Genet Dev Biol. 1982;2(1):65-74

29. Lujan JE, Carlis ME, Lubs HA. A form of X-linked mental retardation with marfanoid habitus. Am J Med Genet. 1984; $17(1): 311-322$

30. Habermann CR, Weiss F, Schoder V, et al. MR evaluation of dural ectasia in Marfan syndrome: reassessment of the established criteria in children, adolescents, and young adults. $R a$ diology. 2005;234(2):535-541 